\title{
Vertical Combdrive Based 2-D Gimbaled Micromirrors With Large Static Rotation by Backside Island Isolation
}

\author{
Sunghoon Kwon, Student Member, IEEE, Veljko Milanović, Member, IEEE, and Luke P. Lee
}

\begin{abstract}
We introduce a backside island isolation method for silicon-on-insulator (SOI)-based microelectromechanical systems technology and demonstrate vertical comb drive-based two-dimensional gimbaled micromirrors with large static rotation using the isolation method. The proposed isolation method provides electrical isolation and mechanical coupling of SOI structures without additional dielectric backfill and planarization by utilizing timed etched backside handle wafer structures. The backside island is a hidden layer beneath the gimbal and allows independent application of actuation potentials to the gimbal and inner mirror. We developed the fabrication process that accommodates the backside island isolation structures into an established vertical comb drive process, thereby allowing implementation of two-axis gimbaled structures. The maximum static optical deflections of the gimbal and mirror are $46^{\circ}$ and $15^{\circ}$, respectively.
\end{abstract}

Index Terms-Backside island isolation, deep reactive ion etching (DRIE), micromirror, raster scanning, silicon-on-insulator (SOI), two-axis scanner, two-dimensional (2-D) scanner, vertical combdrive.

\section{INTRODUCTION}

$\mathbf{S}$ ILICON-ON-INSULATOR (SOI) technology-based microelectromechanical systems (MEMS) micromirrors provide attractive features such as a flat mirror surface, high aspect ratio structures, CMOS compatibility, and relatively simple fabrication [1]. There have been many efforts to employ vertical comb actuators fabricated on SOI wafers [2]-[4]. The high force density of a vertical comb drive actuator enables low voltage and large displacement actuation of these high-aspect SOI structures. However, previous static scanners with SOI vertical comb drives have been limited to single-axis, unidirectional rotation because lower combfingers are defined in and electrically coupled through the handle wafer [3], [4]. For bidirectional one-dimensional (1-D) rotation, the lower comb fingers need to be electrically isolated. Recently, we have developed linearly and independently controllable vertical comb drives using only an SOI device layer [5], [6]. In these vertical comb drives, both upper and lower comb fingers are defined in the SOI layer and are isolated by trenches, resulting

Manuscript received November 4, 2003; revised February 4, 2004. This work was supported by the Defense Advanced Research Projects Agency (DARPA) BioFLIPS program.

S. Kwon and L. P. Lee are with the Berkeley Sensor and Actuator Center, Department of Bioengineering, University of California, Berkeley, CA 94720 USA (e-mail: 1plee@socrates.berkeley.edu).

V. Milanović is with the Adriatic Research Institute, Berkeley, CA 94704-1029 USA.

Digital Object Identifier 10.1109/JSTQE.2004.828493 in bidirectional actuation and large static rotation [6]. This trench isolation method works well for single axis (1-D) applications such as vertical microlens scanners [5] and 1-D micromirrors [6], where either upper or lower comb fingers are anchored to substrate. Our goal was to expand to two-axis [two-dimensional (2-D)] applications.

A gimbaled structure is a common and effective way of implementing rotation around two axes. A two-axis micromirror has been demonstrated by gap closing actuation, which requires linearization of the driving signal and decoupling of crosstalk between each dimension [7], [8]. To implement a two-axis gimbaled micromirror without cross talk between driving voltages, vertical comb based actuation with isolated comb banks are desired. In the two-axis gimbaled structure with vertical combs, two orthogonal vertical comb banks for two-axis actuation are defined in the moving gimbal and need to be electrically isolated for independent actuation of the two axes. This requires electrical isolation as well as mechanical coupling of the isolated comb bank and gimbal. However, trench isolation in SOI wafer does not provide mechanical coupling. Therefore, backfilling an isolation trench with additional deposition of dielectric layer followed by chemical mechanical polishing (CMP) has been used to achieve the mechanical coupling with electrical isolation [9]. However, the additional deposition and the CMP steps add significant complexity and cost. Recently, a thin-film oxide layer has been used to mechanically couple but electrically isolate two laterally movable structures [10]. For out-of-plane actuation, more solid structural support is desired than coupling through a thin-film oxide layer.

In this paper, we introduce an isolation method for SOI MEMS technologies and demonstrate vertical comb drive-based 2-D gimbaled micromirrors with large static rotation based on the isolation method. The developed method, termed backside island isolation, provides electrical isolation as well as mechanical coupling of SOI structures without additional dielectric backfill and planarization. This backside island isolation allows a gimbal structure with electrical isolation, enabling two-axis rotation of micromirror. Large static rotation is achieved by integrating vertical combdrives into the gimbal. A new fabrication process has been developed to combine the backside island isolation with the previously demonstrated vertical combdrive. This paper is organized as follows. First, we describe in Section II the device structure and concept of backside island isolation. In Section III, we present the fabrication of the vertical comb drive-based 2-D micromirror based 


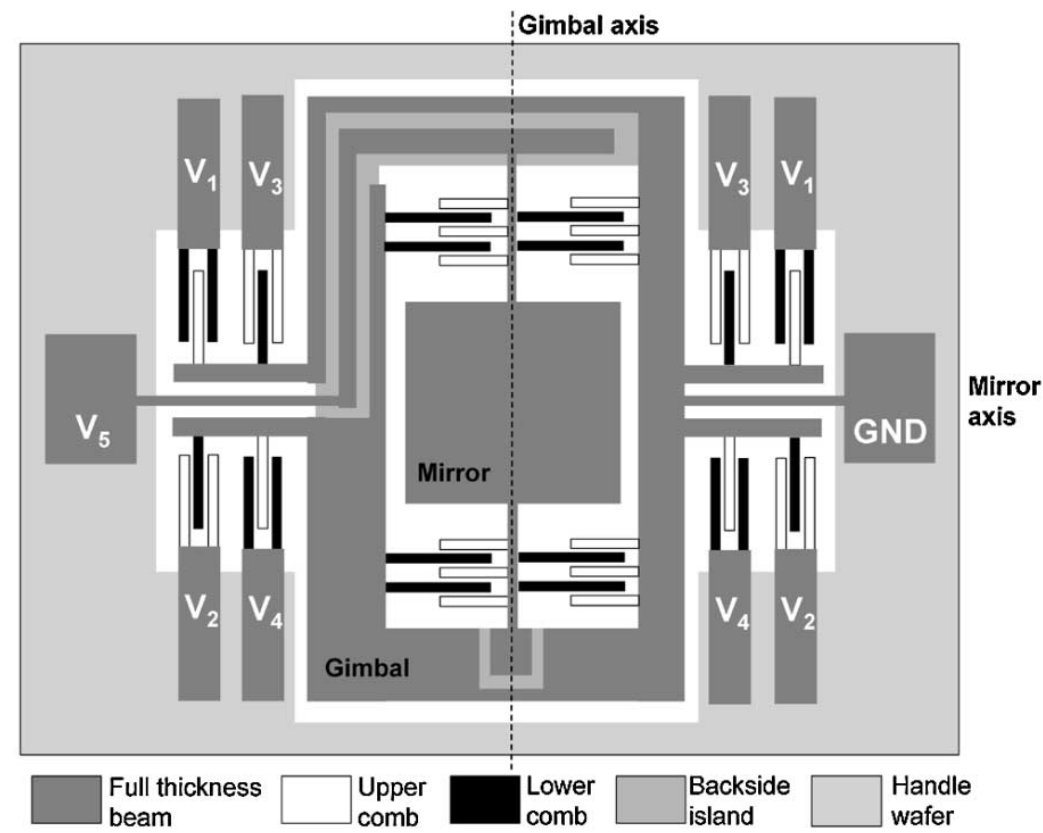

(a)

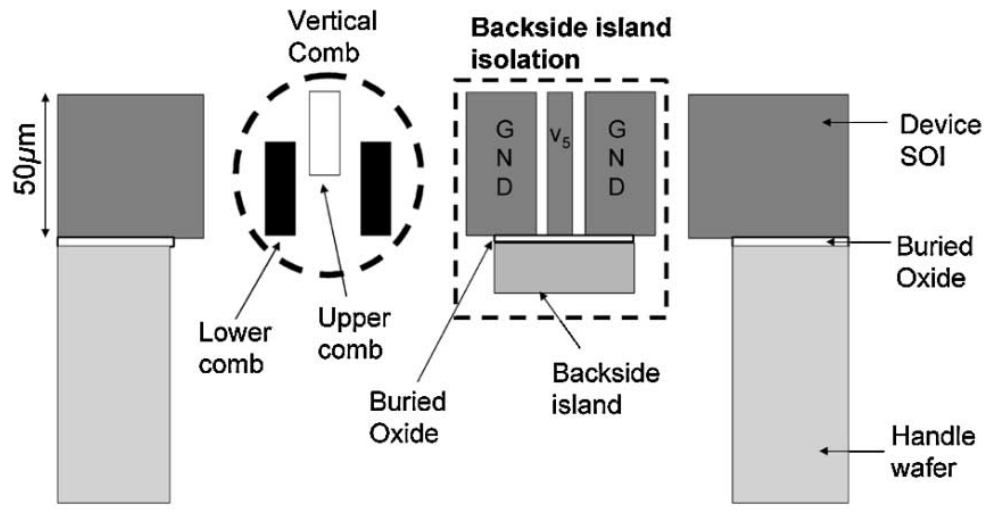

(b)

Fig. 1. (a) Top view of two-axis gimbaled micromirror based on backside island isolation and vertical comb drives. The mirror and gimbal are electrically isolated by trenches but mechanically coupled by the backside island. (b) Cross-sectional view of vertical comb drive and backside island. Note the backside island connects the full thickness SOI beams with different potential. The insulating buried oxide is sandwiched between the SOI layer and backside island.

on the isolation method. Finally in Section IV, we demonstrate performance of the fabricated 2-D micromirror.

\section{DEVICE Sturucture AND BACKSIDE ISLAND ISOLATION}

The design of the two-axis gimbaled micromirror and the cross section of the required beams are shown in Fig. 1(a) and (b), respectively. The 2-D gimbaled mirror design is based on two unique features: the vertical comb drives for actuation and the backside island isolation for coupling, as shown in Fig. 1(a). In the design, micromirror and gimbal are independently actuated by separate vertical comb drives. The independent actuation is achieved by selectively activating separate vertical comb drive banks $\left[V_{1}-V_{5}\right.$, Fig. 1(a)] in the gimbal and mirror. The gimbal axis utilizes four isolated comb drive banks on each side of the rotation axis to achieve bidirectional rotation about the gimbal axis. For example, by actuating electrodes $V_{1}$ and $V_{2}$, rotation in a "positive" direction is achieved, since the comb drive bank with electrode $V_{1}$ pulls the gimbal structure down while the comb drive bank with electrode $V_{2}$ pulls the gimbal
1) Two step backside masking

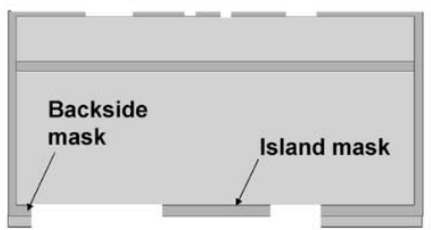

2) Backside DRIE Si/ RIE $\mathrm{SiO}_{2}$

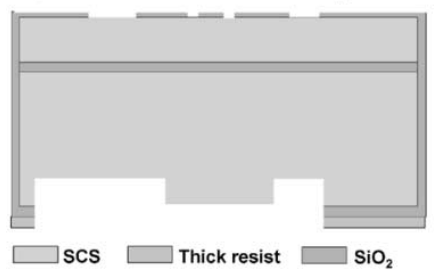

3) Backside blank DRIE Si / RIE $\mathrm{SiO}_{2}$

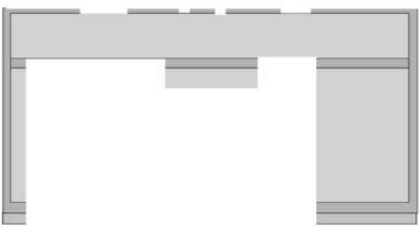

4) Frontside DRIE Si

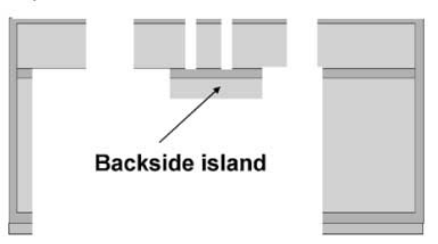

Fig. 2. Backside island process for isolation. The island is formed by two-step DRIE of the substrate.

structure up. Thus, "pure" torque is applied on the suspension beams. To achieve rotation in the opposite direction, electrodes 

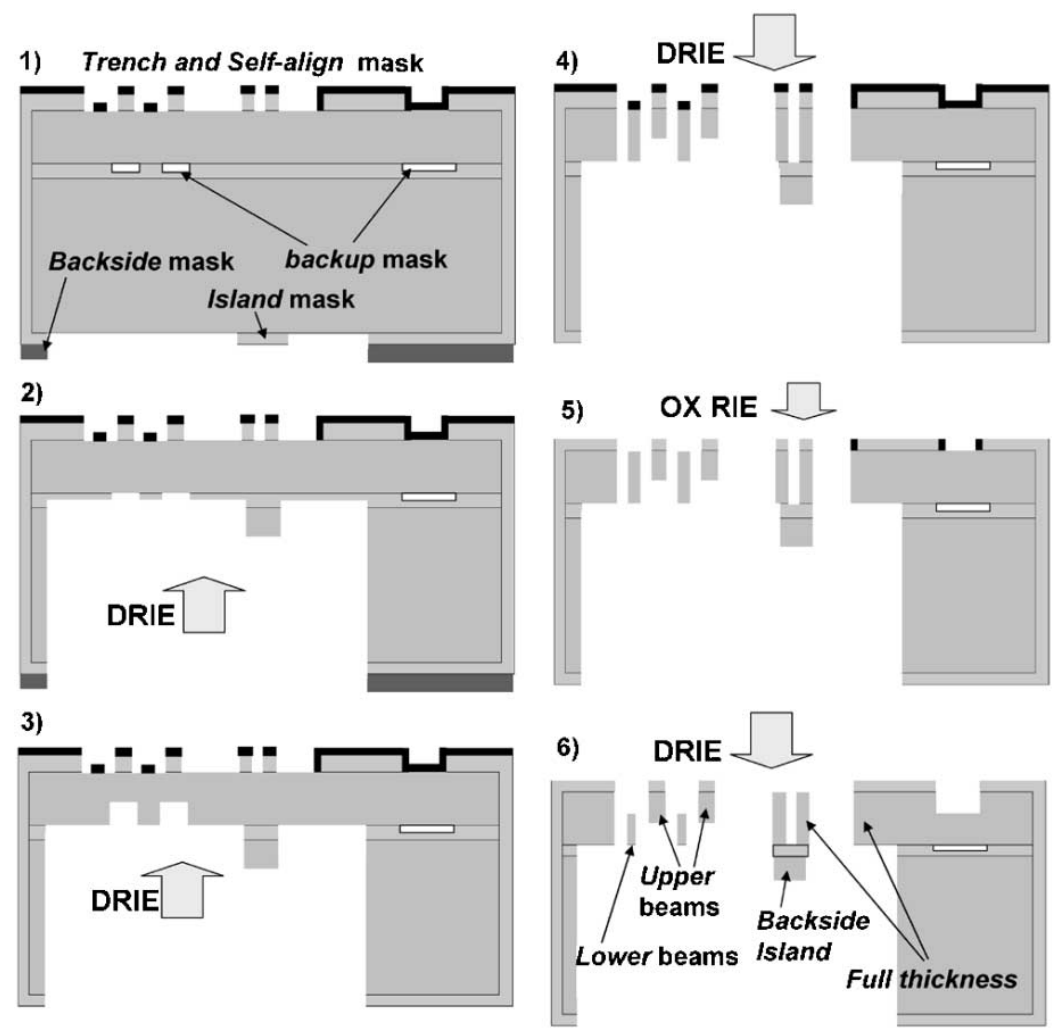

LTO

Wet oxide

Fig. 3. The fabrication process of the vertical comb-based gimbaled micromirror. The backside island is incorporated into the fabrication process for the vertical comb drives.

$V_{3}$ and $V_{4}$ are activated. In all above cases, the reference ground signal [GND, Fig. 1(a)] is applied to the entire gimbal through the right-side torsion flexure.

To achieve rotation of the mirror axis, we require two independent potentials to the comb drive banks along that axis. Without the present isolation technology, this would not be achievable. Namely, the gimbal is grounded (GND) through the right torsion flexure of the gimbal, whereas the potential of mirror $\left(V_{5}\right)$ is applied through the left torsion flexure of the gimbal [Fig. 1(a)]. At the same time, the entire gimbal and mirror need to be mechanically coupled. The isolated coupling is achieved by a backside island below the gimbal structure, as shown in dashed rectangle in Fig. 1(b). The backside island is an $\mathrm{Si}$ island beneath the SOI device layer, which is formed from a timed etch of the handle wafer. The backside island mechanically connects the trench-isolated structures in the SOI and allows for the electrical isolation because the buried oxide layer is sandwiched between the island and SOI structures. In this way, the mirror and gimbal can be mechanically coupled with electrical isolation without dielectric refilling of the isolation trenches.

The thin backside island can be fabricated by two-step deep reactive ion etching (DRIE) from the backside as shown in Fig. 2.

1) Masks are patterned into oxide and a thick photoresist layer.
2) Timed DRIE from the back sets the thickness of the island and the remained island mask is cleaned by reactive ion etching (RIE).

3) Blank DRIE from the back transfer the step height of island until buried oxide is exposed and the buried oxide is cleaned by RIE.

4) Finally, the device structure is defined from the front using DRIE.

The buried oxide layer under the island functions as insulated connector for the top device structure. It should be noted that the process steps for achieving the island do not significantly increase overall process complexity, as the backside etch would be performed for such optical microsystem structures anyway. Namely, the backside etch, which normally requires a single mask, releases large areas for out-of plane motion and rotation of micromirrors. It also provides access to the backside of the device layer for etching of the upper beams. Therefore, to achieve the backside island isolation, only one additional mask is necessary for the backside.

\section{DEVICE FABRICATION}

For a gimbaled two-axis micromirror with vertical comb drives, the backside island process needs to be incorporated with the fabrication process for vertical comb drives. Fig. 3 shows the fabrication process for the gimbaled micromirror. 
(a)
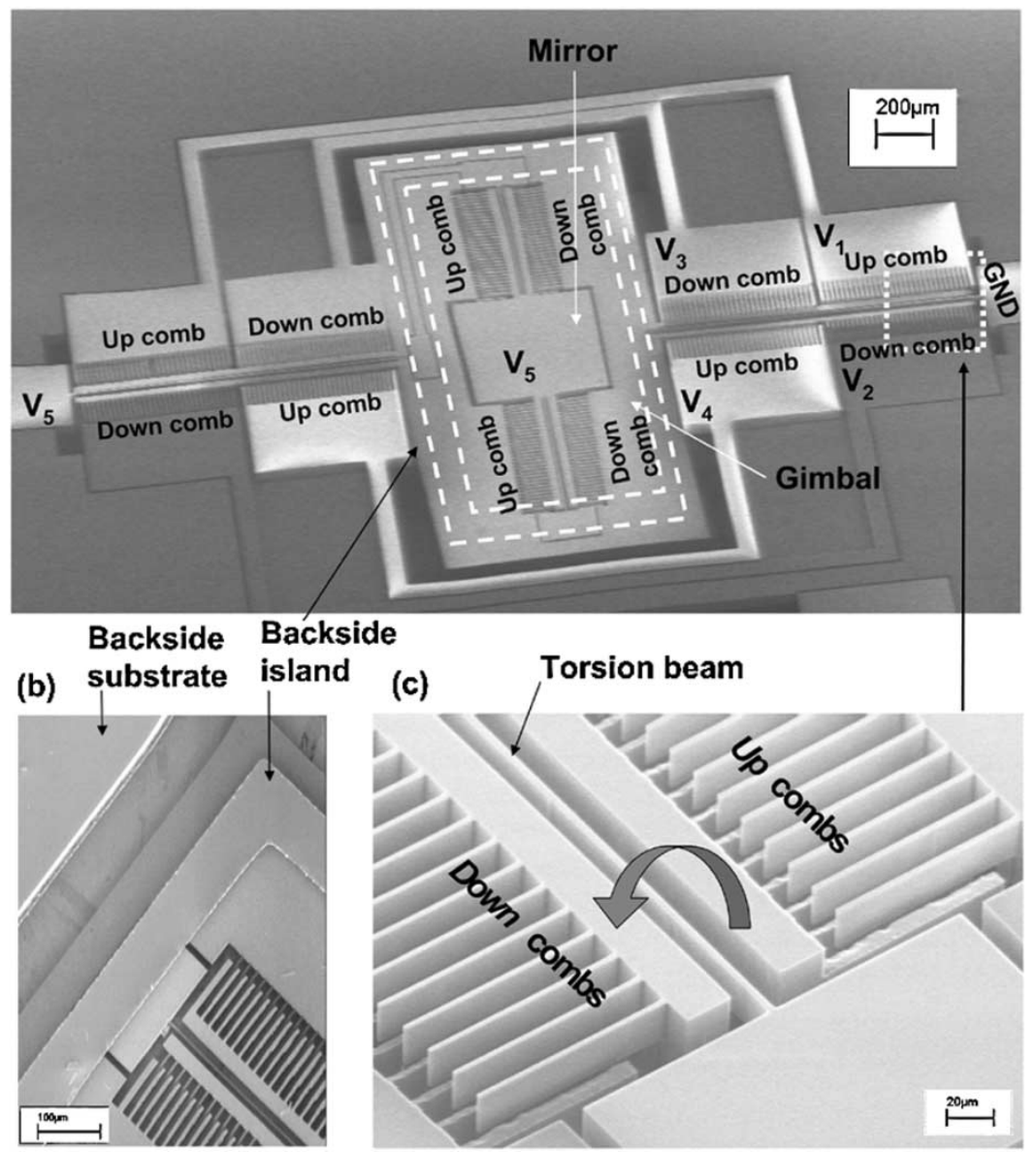

Fig. 4. (a) SEM of two-axis gimbaled micromirror using SOI (top view). (b) SEM of the backside island (bottom view). The mirror is anchored on the island. (c) SEM of vertical combdrives and torsion beam from typical devices.

\section{A. Mask Layer Preparation}

The process begins by fabricating the 4-in SOI wafers. One wafer, intended for the SOI handle, is double side polished with a thickness of $300 \pm 1 \mu \mathrm{m}$. The second wafer, which is to become the device layer, is an $n$-type wafer, standard thickness $(525 \pm 25) \mu \mathrm{m}$, and is single-side polished. A wet thermal oxide of $1 \mu \mathrm{m}$ is grown on both wafers. The oxide on the handle wafer's side is patterned before the bonding. Namely, after a $1-\mu \mathrm{m}$ thermal oxide is grown on both wafers, the wafer intended for SOI handle is patterned with the mask backup (Fig. 3) and the oxide is etched down to the silicon. After removing the photoresist mask and cleaning, the wafers are prebonded, annealed, and ground and polished to the desired device layer thickness.

On the finished SOI wafers, the two front-side masks are prepared utilizing oxides of two thicknesses. The mask preparation is arranged to provide self-alignment of vertical combdrives [11]. Due to the fact that the backup mask is already buried within the SOI wafer, the mask preparation process requires that both front-side masks be aligned to that buried layer. On the backside of the wafer, two additional masks are employed and aligned to the front-side features. The masks for Backside and Island are patterned on the $1.5 \mu \mathrm{m}$ of oxide from front-side preparation and an additional 9- $\mu \mathrm{m}$-thick photoresist layer, respectively. These two masks are for generating two different step heights in the next DRIE steps. To realize the backside island isolation, the thickness of each mask layer should be carefully determined based on the desired thickness of the backside island and the etching selectivity between oxide, silicon and photoresist. In other words, the oxide backside mask should be thick enough to etch through the wafer; however, at the same time, the residual of oxide island mask should be able to easily etched away after the first backside DRIE for silicon. The following inequality should be satisfied when we determine the thickness of the mask layers:

$$
\begin{aligned}
T_{\mathrm{ox}}\left(S_{\mathrm{Si}: 0 x}-\frac{S_{\mathrm{Si}: \mathrm{PR}}}{S_{\mathrm{Ox}: \mathrm{PR}}}\right) & +S_{\mathrm{Si}: \mathrm{PR}} \cdot T_{\mathrm{PR}} \\
& +\frac{S_{\mathrm{Si}: \mathrm{PR}}}{S_{\mathrm{Si}: \mathrm{Ox}} \cdot S_{\mathrm{Ox}: \mathrm{PR}}} \cdot T_{\mathrm{BI}}>T_{w}
\end{aligned}
$$

where $T_{\mathrm{ox}}, T_{\mathrm{PR}}, T_{\mathrm{BI}}$, and $T_{w}$ denote the thickness of the oxide mask layer, the thick resist, backside island, and handle wafer, respectively. $S_{\mathrm{Si}: \mathrm{Ox}}, S_{\mathrm{Si}: \mathrm{PR}}$, and $S_{\mathrm{Ox}: \mathrm{PR}}$ represent the dry etching selectivity of silicon to oxide, silicon to photoresist, and oxide to photoresist, respectively. The left side of the inequality is the maximum etchable depth of the backside opening at given mask layer thicknesses in the backside island process. For example, typical values of $S_{\mathrm{Si}: \mathrm{Ox}}, S_{\mathrm{Si}: \mathrm{PR}}, S_{\mathrm{Ox}: \mathrm{PR}}$ are 100 , 50 , and 2 , respectively. If the desired thickness of the backside 


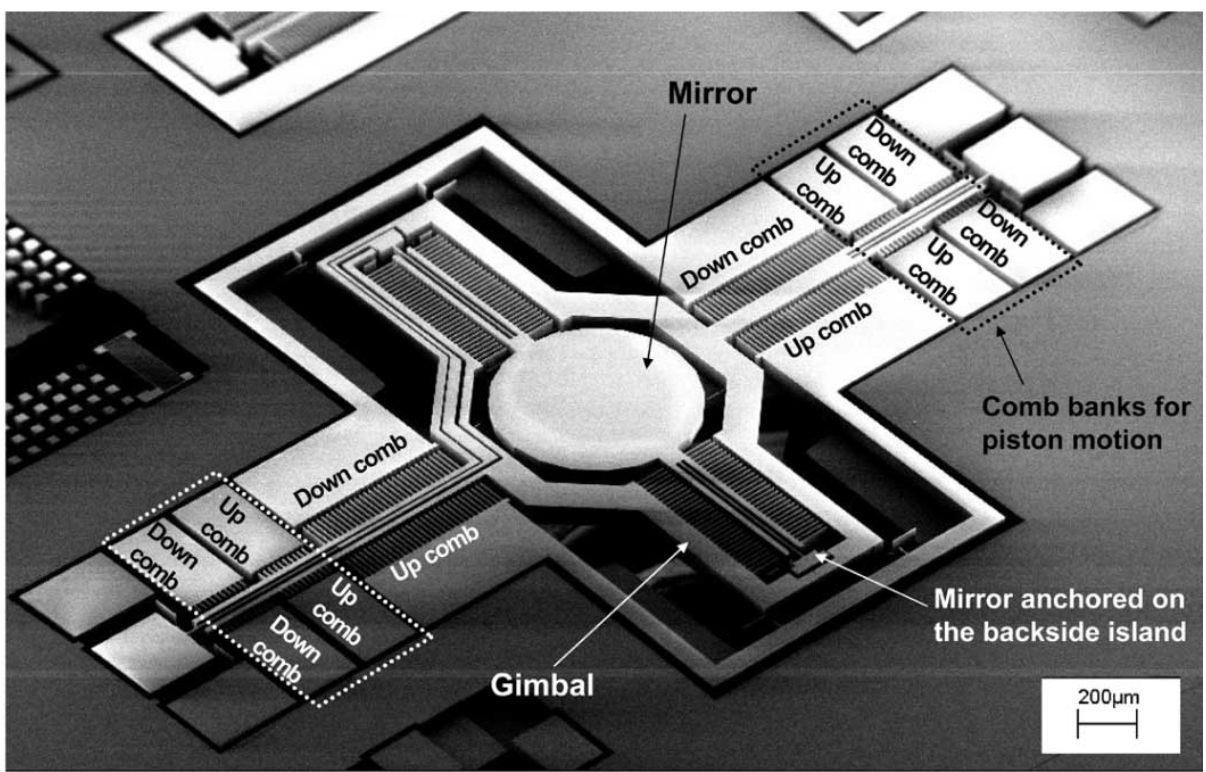

Fig. 5. SEM of second version of 2-D gimbaled micromirror. The gimbal is minimized and electrodes for piston motion of the micromirror are added.

island $T_{\mathrm{BI}}$ is $50 \mu \mathrm{m}$ and the handle wafer thickness $T_{w}$ is $500 \mu \mathrm{m}$, inequality (1) will become $\left(75 T_{\mathrm{ox}}+50 T_{\mathrm{PR}}>487.5\right)$. Therefore, a $1.5-\mu \mathrm{m}$-thick oxide layer and $9-\mu \mathrm{m}$-thick photoresist layer will ensure a 562- $\mu \mathrm{m}$ maximum etchable depth of the backside opening, resulting in enough process margin for backside opening.

\section{B. Backside DRIE for Island}

The backside island is fabricated by DRIE etching from the back. The concept of this backside etching is described in the previous section. The first backside DRIE is timed and determines the thickness of the backside island. After the timed etch, the island mask is removed by RIE. Next, backside DRIE is performed until the etched trench reaches the buried oxide.

\section{Backside DRIE for Upper Comb Fingers}

This exposes the insulating oxide and the buried backup mask [Fig. 4(b)]. The insulating oxide is then thinned (by timed oxide etch) $\sim 1.2 \mu \mathrm{m}$, which exposes the backside of the device silicon layer in the patterned areas of the buried backup mask. The final backside DRIE step shown is to perform the actual Backup DRIE into the device layer. This etch is timed to leave a desired thickness of upper beams. In most cases, we etched about $20 \mu \mathrm{m}$ of device layer silicon such that the remaining upper beam thickness would be $\sim 30 \mu \mathrm{m}$. Lastly, the insulating oxide is fully removed from the backside.

\section{Frontside DRIE}

The front-side DRIE steps are shown in Fig. 3(4) and Fig. 3(5) to better understand the formation of vertical comb drives. First, DRIE etches through the device layer as shown in Fig. 3(4). Then, oxide plasma etch of $\sim 0.8 \mu \mathrm{m}$ on the front side thins down oxide everywhere, removing the thinner oxide mask [Fig. 3(5)]. The second and final DRIE is performed until the lower beams are lowered to the desired height of $30 \mu \mathrm{m}$. The final result is shown in the schematic in Fig. 3(6).

\section{RESULTS}

Scanning electron microscope (SEM) images of the fabricated device are shown in Fig. 4. The mirror, gimbal, and vertical comb drives are shown in Fig. 4(a). The configuration of vertical comb banks allows bidirectional rotation of the gimbal and unidirectional rotation of the mirror. The dashed box in Fig. 4(a) represents the location of the backside island, which is hidden beneath the gimbal. Fig. 4(b) is an SEM of the backside island taken from below. The backside island is attached underneath the gimbal and the mirror is anchored to the backside island. The buried oxide is sandwiched between the mirror and the backside island, allowing the mirror to be electrically isolated. Also the mirror and gimbal are isolated by trenches around the mirror. In this way, different actuation voltages can be applied as shown in the Fig. 4(a). The vertical comb banks and the torsion beam in the gimbal from a similar device are shown in Fig. 4(c). The up combs and down comb will pull up and push down the gimbal, resulting in counterclockwise (CCW) rotation. Another micromirror with reduced gimbal area and piston motion capability is shown in Fig. 5. Instead of the electrodes for bidirectional actuation of the outer gimbal, electrodes for piston motion are added to the device. Piston actuation (pure vertical actuation) ranging from -7 to $+7 \mu \mathrm{m}$ has been demonstrated with a voltage smaller than $100 \mathrm{~V}$. The isolation trench that isolates the mirror and gimbal is clearly shown in the figure. The mirror potential $\left(V_{5}\right)$ is applied through one of the gimbal's torsion flexures.

Fig. 6 shows typical experimental dc deflection characteristics for the gimbal and mirror. The gimbal can be rotated from $-23^{\circ}$ to $23^{\circ}$, resulting in a maximum optical static scan angle of $46^{\circ}$. Both clockwise (CW) and CCW rotation has been demonstrated. Activation of electrode $V_{1}$ and $V_{2}$ results in $\mathrm{CW}$ rotation of the gimbal and activation of $V_{3}$, and $V_{4}$ rotates the gimbal in $\mathrm{CCW}$ direction. The maximum static scan angle for the mirror is $15^{\circ}$ as shown in Fig. 6. This bidirectional actuation of the gimbal results from the isolated vertical comb banks. Conversely, the 


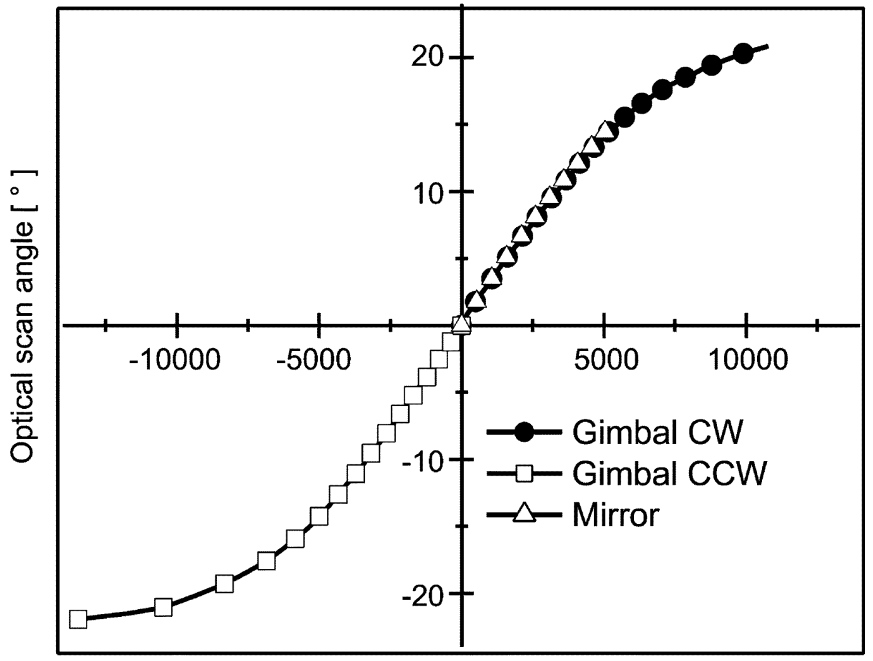

(Voltage sign $\left.{ }^{*}\right)($ Voltage squared $)\left[\mathrm{V}^{2}\right]$

* Voltage sign is positive for the voltage applied to electrode $V_{1}$ and $V_{2}$, and is negative for the voltage applied to electrode $V_{3}$ and $V_{4}$.

Fig. 6. The static actuation characteristic of the gimbaled micromirror The gimbal can be actuated in both $\mathrm{CW}$ and $\mathrm{CCW}$ directions.

TABLE I

SUMMARY OF ACTUATION CHARACTERISTIC OF THE GIMBALED MiRROR

\begin{tabular}{c|c|c|c|c}
\hline $\begin{array}{c}\text { Mirror } \\
\text { elements }\end{array}$ & $\begin{array}{c}\text { Resonant } \\
\text { Frequency } \\
{[\mathrm{Hz}]}\end{array}$ & $\mathbf{Q}$ & $\begin{array}{c}\text { Maximum static } \\
\text { optical scanning } \\
\left.\text { angle [ }{ }^{\circ}\right]\end{array}$ & Actuation direction \\
\hline Mirror & 2248 & 58 & 15 & Uni-direction \\
\hline Gimbal & 182 & 28 & 46 & Bi-direction \\
\hline
\end{tabular}
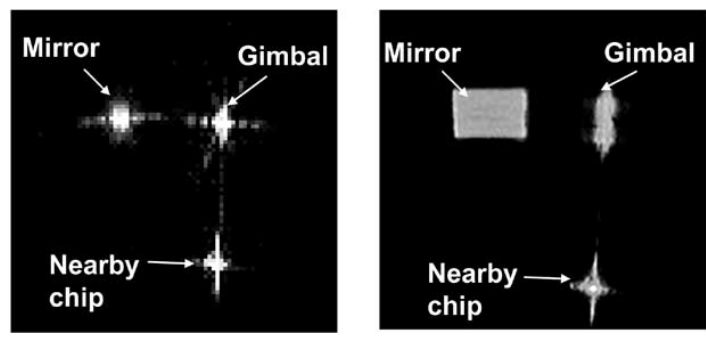

Fig. 7. Demonstration of static 2-D steering (left) and raster scanning (right) of the laser beam. The label denotes the structures that each laser beam is reflected from.

static rotation of the mirror is limited to unidirectional actuation because only two separate electrical connections from the outside gimbal to the structures inside the gimbal are allowed through the flexure of the gimbal. Since one side of the flexure is already used to control the mirror potential, the potential of the entire gimbal is controlled via the other side of the torsion flexure. Therefore, the comb drives in the entire gimbal cannot be selectively activated for bidirectional rotation. The typical mirror mechanical characteristics are shown in Table I. The resonant frequency of the mirror can be designed to be higher. The penalty of using the backside island is increased mass of the gimbal. But this is durable for raster scanning application because the gimbal is usually actuated in off-resonance. Demonstration of laser beam scanning is shown in Fig. 7. The label denotes the structures from which a laser beam is reflected. In this experiment, the mirror and gimbal deflect the laser beam in the horizontal and vertical direction, respectively. The spots in Fig. 7(a) are from dc static actuation. The rectangular beam scanning pattern in Fig. 7(b) is from raster scanning that is generated from resonating mirror at $2.2 \mathrm{kHz}$ and off-resonance actuation of the gimbal at $30 \mathrm{~Hz}$.

\section{CONCLUSION}

We describe a backside isolation method for SOI-based MEMS technologies and demonstrate vertical comb drive-based 2-D gimbaled micromirrors with large static rotation using the isolation method. The backside island isolation not only achieves electrical isolation and mechanical coupling of SOI structures without additional dielectric backfill and planarization but also provides an additional structural layer by utilizing an etched handle wafer as a hidden device layer. Incorporation of the backside island isolation method into the previously demonstrated vertical comb drive has been demonstrated.

\section{ACKNOWLEDGMENT}

The authors would like to thank G. Matus for his assistance with experiments and helpful discussions.

\section{REFERENCES}

[1] S. Blackstone and T. Brosnihan, "SOI MEMS technologies for optical switching," in Proc. IEEE/LEOS Int. Conf. Optical MEMS, 2001, pp. 35-36.

[2] J. L. Yeh, H. Jiang, and N. C. Tien, "Integrated polysilicon and DRIE bulk silicon micromachining for an electrostatic torsional actuator," Journal of Microelectromechanical Systems, vol. 8, pp. 456-465, 1999.

[3] R. A. Conant, J. T. Nee, K. Y. Lau, and R. S. Muller, "A flat high-frequency scanning micromirror," in Prof. IEEE Solid-State Sensor and Actuator Workshop, Hilton Head Island, SC, USA, 2000, pp. 6-9.

[4] U. Krishnamoorthy, L. Daesung, and O. Solgaard, "Self-aligned vertical electrostatic combdrives for micromirror actuation," J. Microelectromech. Syst., vol. 12, pp. 458-464, Aug. 2003.

[5] S. Kwon, V. Milanovic, and L. P. Lee, "Large-displacement vertical microlens scanner with low driving voltage," IEEE Photon. Technol. Lett., vol. 14, pp. 1572-1574, Nov. 2002.

[6] V. Milanovic, S. Kwon, and L. P. Lee, "Monolithic vertical combdrive actuators for adaptive optics," in Proc. 2002 IEEE/LEOS Int. Conf. Optical MEMS, pp. 57-58.

[7] G. D. J. Su, H. Toshiyoshi, and M. C. Wu, "Surface-micromachined 2-D optical scanners with high-performance single-crystalline silicon micromirrors," IEEE Photon. Technol. Lett., vol. 13, pp. 606-608, June 2001.

[8] H. Toshiyoshi, W. Piyawattanametha, C.-T. Chan, and M. C. Wu, "Linearization of electostatically actuated micromachined 2D optical scanner," J. Microelectromech. Syst., vol. 10, pp. 205-214, June 2001.

[9] H. Schenk, P. Durr, D. Kunze, H. Lakner, and H. Kuck, "A resonantly excited 2D-micro-scanning-mirror with large deflection," Sens. Actuators A, Phys., vol. 89, pp. 104-111, 2001.

[10] C. Tsai, P. A. Stupar, R. L. Borwick III, M. Pai, and J. DeNatale, “An isolated tunable capacitor with a linear capacitance voltage behavior," in Transducers '03, 12th Int. Conf. Solid-State Sensors, Actuators and Microsystems, Thousand Oaks, CA, June 8-12, 2003, pp. 833-836.

[11] V. Milanovic, "Multilevel-beam SOI MEMS for optical applications," in Proc. 9th IEEE Int. Conf. Electronics, Circuits and Systems (ICECS'02), 2002, pp. 281-215. 


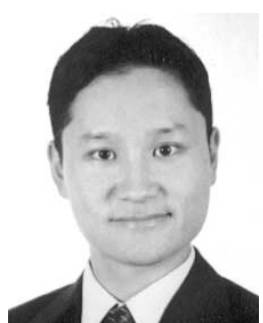

Sunghoon Kwon (S'98) received the B.S. degree in electrical engineering and computer science and the M.S. degree in biomedical engineering from Seoul National University (SNU), Seoul, Korea, in 1998 and 2000, respectively. His master's research was focused on human computer interface using bioelectric signals. He is currently working toward the $\mathrm{Ph} . \mathrm{D}$. degree in bioengineering at the University of California, Berkeley, on the topic of MEMS laser scanning confocal microscopes for micro total analysis systems.

$\mathrm{He}$ is an Affiliate of the Berkeley Sensor and Actuator Center, Berkeley. His research interests include miniaturized photonic systems, nanostructures for biosensing, handheld medical instruments, and human-computer interface.

Mr. Kwon is a Student Member of EMBS and SPIE.

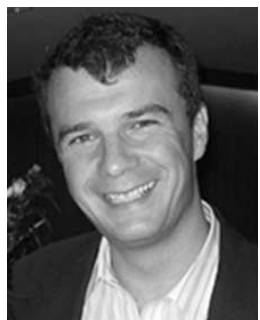

Veljko Milanović (S'92-M'99) received the M.Sc. and D.Sc. degrees in electrical engineering-microelectronics from George Washington University, Washington, DC, in 1996 and 1998, respectively.

He was a Postdoctoral Researcher at the Berkeley Sensor and Actuator Center and a Guest Researcher in the Semiconductor Electronics Division of the National Institute of Standards and Technology. He founded the Adriatic Research Institute, Berkeley, $\mathrm{CA}$, with a focus on microsystems and nanosystems research and education for public benefit. He is also currently involved in biomolecular sciences research in the Nanoengineering Laboratory at the Mechanical Engineering Department, University of California, Berkeley. He has been involved in MEMS and nanotechnology research and development efforts for close to ten years.

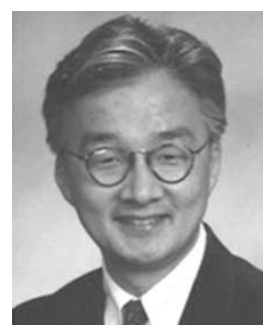

Luke P. Lee received the B.A. degree in biophysics and the Ph.D. degree in applied physics (with a minor in bioengineering) from the University of California, Berkeley (UC Berkeley).

$\mathrm{He}$ is Assistant Professor in the Department of Bioengineering and Co-Director of the Berkeley Sensor and Actuator Center, UC Berkeley. He has more than ten years of industrial experience in microphotonic devices, holography, superconducting quantum interference devices (SQUIDs), and magnetic bioassays. He has authored or coauthored over 60 journal and conference articles on integrated surface emitting laser diodes, SQUIDs, biomagnetic sensors, biophotonic MEMS, nanogap electrode junctions for label-free DNA, and protein detection. His current research interests are integrated biophotonic devices, nanogap biomolecular junctions, neural interfaces, Bio-Polymer-Opto-Electro-Mechanical-Systems (BioPOEMS) for lab-on-a-chip, nanophotonics for single molecule detection, and cellular pathway monitoring. 